\title{
Optimized sixth-order monotonicity-preserving scheme by nonlinear spectral analysis
}

\author{
Xin-liang $\mathrm{Li}^{*}{ }^{\dagger}$, Yan Leng and Zhi-wei He \\ LHD Institute of Mechanics, CAS, Beijing 100190, China
}

\begin{abstract}
SUMMARY
In this paper, sixth-order monotonicity-preserving optimized scheme (OMP6) for the numerical solution of conservation laws is developed on the basis of the dispersion and dissipation optimization and monotonicitypreserving technique. The nonlinear spectral analysis method is developed and is used for the purpose of minimizing the dispersion errors and controlling the dissipation errors. The new scheme (OMP6) is simple in expression and is easy for use in CFD codes. The suitability and accuracy of this new scheme have been tested through a set of one-dimensional, two-dimensional, and three-dimensional tests, including the one-dimensional Shu-Osher problem, the two-dimensional double Mach reflection, and the RayleighTaylor instability problem, and the three-dimensional direct numerical simulation of decaying compressible isotropic turbulence. All numerical tests show that the new scheme has robust shock capturing capability and high resolution for the small-scale waves due to fewer numerical dispersion and dissipation errors. Moreover, the new scheme has higher computational efficiency than the well-used WENO schemes. Copyright (C) 2013 John Wiley \& Sons, Ltd.
\end{abstract}

Received 18 November 2012; Revised 7 March 2013; Accepted 25 April 2013

KEY WORDS: nonlinear spectral analysis; monotonicity-preserving; optimization; shock-capturing schemes; dissipation; dispersion

\section{INTRODUCTION}

High order shock capturing schemes play important roles in the detailed simulations (DNS or LES) of supersonic or hypersonic complex flows, and to develop high resolution shock capturing scheme is a hot topic in the recent CFD research. Unlike the incompressible or subsonic turbulent flows, shocks or shocklets often appear in supersonic/hypersonic turbulent flows, and those shock waves pose severe challenges to DNS or LES in supersonic/hypersonic turbulent flows [1]. To resolve the small-scale fluctuation, the scheme must have low dissipation and low dispersion, but with non-dissipative schemes, the nonphysical oscillations near shock waves will cause, in many cases, appearance of negative temperatures or pressures in the flows when turbulent Mach number is high enough. Therefore, schemes for compressible turbulent flows require robust shock capturing capability, as well as minimized dissipation and dispersion errors.

Currently, high order WENO schemes [2,3] are well used in the DNS/LES of supersonic/hypersonic turbulent flows for their high robustness and accuracy. However, WENO schemes are computationally expensive $[4,5]$ and in some cases somewhat too dissipative for small scales [1]. A lot of efforts have been devoted to the lower dissipation properties of WENO schemes, such as hybrid schemes [6-10] or optimized WENO schemes [11,12].

Suresh and Huynh [5] developed a class of monotonicity-preserving schemes (MP schemes), which have very high resolution for small scales and are more efficient than the mostly used WENO schemes. An MP scheme contains a linear (high order) part and an MP limiter part. Suresh and

*Correspondence to: Xin-liang Li, LHD Institute of Mechanics, CAS, Beijing-100190 China.

†E-mail: lixl@imech.ac.cn 
Huynh's research mainly focus on the improvement of the MP limiter, and the linear part of MP scheme is less studied. In Suresh and Huynh's research, an ordinary fifth-order or seventh-order upwind biasing schemes are suggested as the linear part of MP schemes. The authors studied the MP schemes based on the nonlinear spectrum analysis for the dispersion and dissipation, and found that the linear part of MP scheme also has great effects on the total performance of the whole scheme. In the low wave number range (smooth field), the limiter of MP scheme does not work, and the linear part dominates the performance of the whole scheme. However, even in high wave number range (near the shock or dramatic disturbance region), the linear part also has great effects on the performance of whole scheme. So, the given suitable linear part is also very important for MP schemes. On the basis of the dispersion and dissipation optimization [13,14], by using nonlinear spectrum analysis and monotonicity-preserving technique [5], a sixth-order optimized MP scheme (OMP6) is proposed.

Numerical tests show that the new scheme (OMP6) is robust enough for supersonic/hypersonic flow and have very high computational efficiency. And, the tests also show that OMP6 is less dissipative than classical seventh-order WENO scheme (WENO7) [3] or original seventh-order MP scheme [5].

The paper is organized as follows. Section 2 focuses on the construction of OMP6. In Section 3 , the new scheme is extended to the Euler equations. Section 4 is devoted to numerical results, and comparisons are made with the classical seventh-order WENO scheme (WENO7) and original seventh-order MP scheme (MP7).

\section{DESCRIPTION OF THE NUMERICAL SCHEME}

Consider the scalar hyperbolic conservation law given by

$$
\frac{\partial u}{\partial t}+\frac{\partial f(u)}{\partial x}=0
$$

Simplicity, let

$$
f(u)=a u, \quad a>0
$$

The semi-discrete scheme of (2.1) is

$$
\frac{\partial u}{\partial t}+\frac{1}{\Delta x}\left(f_{j+1 / 2}-f_{j-1 / 2}\right)=0
$$

where $\Delta x$ is the grid spacing, and $f_{j+1 / 2}$ is the numerical flux, which is given by numerical scheme. We will construct the sixth-order optimized MP scheme (OMP6) in this section.

\subsection{Construction of linear scheme with free parameters}

As mentioned earlier, the construction of sixth-order optimized MP scheme (OMP6) begins with a linear scheme with free parameters, and then the nonlinear limiter is added for shock capturing. Finally, the free parameters are set by optimization based on nonlinear spectrum analysis.

The linear scheme for numerical flux $f_{j+1 / 2}^{\text {Linear }}$ is constructed in eight points stencil $\left[x_{j-3}, \ldots \ldots, x_{j+3}, x_{j+4}\right]$ (Figure 1$)$ as

$$
f_{j+1 / 2}^{\text {Linear }}=\sum_{k=1}^{8} a_{k} f_{j+k-4}
$$

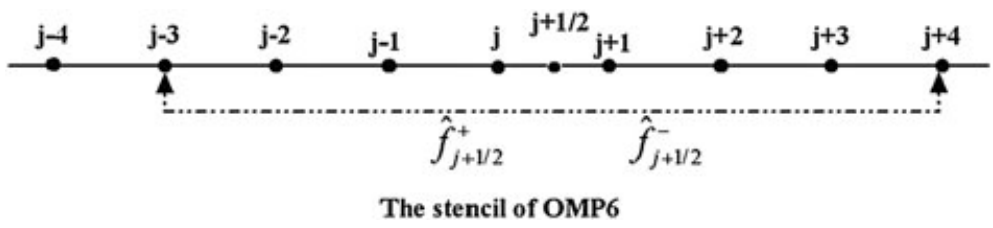

Figure 1. Stencils for the construction of $f_{j+1 / 2}$. 
Clearly, on eight points stencil, we can construct the highest eighth-order both linear central and upwind schemes. Here, we require that the linear scheme has sixth-order accuracy, then the Taylor expansion gives six constrains for accuracy condition, so, there should be two free parameters in the expression of coefficients $\left\{a_{k}\right\}$. As addressed in [10], the dissipation and dispersion property of the linear scheme can be determined by the two free parameters separately, that is, one parameter determines the dissipation, and another parameter determines the dispersion.

Let

$$
a_{1}=-\frac{\xi-\eta}{2}, a_{8}=\frac{\xi+\eta}{2}
$$

where $\xi$ and $\eta$ are the two free parameters. By solving the linear equations of accuracy constrains from Taylor expansion, we can obtain $\left\{a_{k}\right\}$. Then, the linear scheme with free parameters (2.3) can be rewritten as

$$
\begin{aligned}
f_{j+1 / 2}^{\text {Linear }}= & \frac{\xi+\eta}{2} f_{j+4}+\left(\frac{1}{60}-\frac{7 \xi+5 \eta}{2}\right) f_{j+3}+\left(-\frac{2}{15}+\frac{21 \xi+9 \eta}{2}\right) f_{j+2}+\left(\frac{37}{60}-\frac{35 \xi+5 \eta}{2}\right) f_{j+1} \\
& +\left(\frac{37}{60}+\frac{35 \xi-5 \eta}{2}\right) f_{j}+\left(-\frac{2}{15}-\frac{21 \xi-9 \eta}{2}\right) f_{j-1}+\left(\frac{1}{60}+\frac{7 \xi-5 \eta}{2}\right) f_{j-2}-\frac{\xi-\eta}{2} f_{j-3}
\end{aligned}
$$

Because meeting the accuracy is constrained, (2.4) has sixth-order accuracy.

By using Fourier analysis [13], the modified wave number $\tilde{k}=k_{r}+i k_{i}$ of the linear scheme (2.4) can be given. The real part $k_{r}$ is related to the dissipation error, and the image part $k_{i}$ is related to the dispersion error of the scheme. The expressions are

$$
\begin{aligned}
& k_{r}=8 \xi(\cos (\alpha)-1)^{4} \\
& k_{i}=\eta \sin (4 \alpha)+\left(\frac{1}{30}-6 \eta\right) \sin (3 \alpha)+\left(14 \eta-\frac{3}{10}\right) \sin (2 \alpha)+\left(\frac{3}{2}-14 \eta\right) \sin (\alpha)
\end{aligned}
$$

where $\alpha=k \Delta x$ is the scaled wave number. Eq. (2.5) shows that the dissipation of linear scheme (2.4) is only related with free parameter $\xi$, and the dispersion is only related with $\eta$. This means that the dissipation and dispersion properties of linear scheme (2.4) can be optimized independently. Particularly, when $\xi=0 ; \eta=-\frac{1}{140}$, scheme (2.4) is an eight-order central scheme.

\subsection{Improving shock capturing ability by adding monotonicity-preserving limiter [5]}

Following the MP technique of Suresh and Huynh [5], MP limiter is added to the linear scheme (2.4) to improve the shock capturing capability. The linear flux calculated by (2.4) is called original flux. For the limiting procedure, it is of critical importance that it should not alter the original flux in smooth regions, so, the accuracy is preserved. Limiter takes effect and pulls the original flux into a certain interval near a discontinuity; as a result, monotonicity is preserved. After adding limiter into the linear scheme (2.4), the MP schemes with free parameters (MP-FP) are constructed. The MP-FP schemes are as follows:

$$
f_{j+1 / 2}=\left\{\begin{array}{lrr}
f_{j+1 / 2}^{\text {Linear }} \quad \text { if } \quad\left(f_{j+1 / 2}^{\text {Linear }}-f_{j}\right)\left(f_{j+1 / 2}^{\text {Linear }}-f_{j+1 / 2}^{\text {MP }}\right) \leqslant \varepsilon & \left(\varepsilon=10^{-10}\right) \\
f_{j+1 / 2}^{\text {Linear }}+\operatorname{minmod}\left(f_{j+1 / 2}^{\text {min }}-f_{j+1 / 2}^{\text {Linear }}, f_{j+1 / 2}^{\text {max }}-f_{j+1 / 2}^{\text {Linear }}\right) & \text { otherwise }
\end{array}\right.
$$

where

$$
\begin{aligned}
f_{j+1 / 2}^{\mathrm{MP}} & =f_{j+1 / 2}^{\mathrm{Linear}}+\operatorname{minmod}\left[\left(f_{j+1}-f_{j}\right), 4\left(f_{j}-f_{j-1}\right)\right] \\
f_{j+1 / 2}^{\mathrm{max}} & =\min \left[\max \left(f_{j}, f_{j+1}, f_{j+1 / 2}^{\mathrm{MD}}\right), \max \left(f_{j}, f_{j+1 / 2}^{\mathrm{UL}}, f_{j+1 / 2}^{\mathrm{LC}}\right)\right] \\
f_{j+12}^{\min } & =\max \left[\min \left(f_{j}, f_{j+1}, f_{j+1 / 2}^{\mathrm{MD}}\right), \min \left(f_{j}, f_{j+1 / 2}^{\mathrm{UL}}, f_{j+1 / 2}^{\mathrm{LC}}\right)\right] \\
f_{j+1 / 2}^{\mathrm{UL}} & =f_{j}+4\left(f_{j}-f_{j-1}\right) ; f_{j+1 / 2}^{\mathrm{MD}}=\frac{1}{2}\left(f_{j}+f_{j+1}\right)-\frac{1}{2} d_{j+1 / 2}^{\mathrm{M}} ; \\
f_{j+1 / 2}^{\mathrm{LC}} & =\frac{1}{2}\left(3 f_{j}-f_{j-1}\right)+\frac{4}{3} d_{j-1 / 2}^{\mathrm{M}} \\
d_{j+1 / 2}^{\mathrm{M}} & =\operatorname{minmod}\left(4 d_{j}-d_{j+1}, 4 d_{j+1}-d_{j}, d_{j}, d_{j+1}\right) ; d_{j}=f_{j-1}+f_{j+1}-2 f_{j},
\end{aligned}
$$

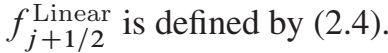


Condition $\left(f_{j+1 / 2}^{\text {Linear }}-f_{j}\right)\left(f_{j+1 / 2}^{\text {Linear }}-f_{j+1 / 2}^{\mathrm{MP}}\right) \leqslant \varepsilon$ determines the smoothness of the stencil. If it holds, the region is smooth, and there is no change with the original flux, otherwise, the limiting procedure takes effort for the discontinuous region. The definition of the minmod function and details of the MP technique can be consulted in ref. [5].

Now, the nonlinear scheme has been constructed by adding MP limiter. In the following, the free parameters $\xi, \eta$ in $f_{j+1 / 2}^{\text {Linear }}$ will be determined by nonlinear spectral analysis.

\subsection{Optimization for dissipation and dispersion by nonlinear spectral analysis}

2.3.1. Nonlinear spectral analysis. Pirozzoli studied the performance of shock capturing schemes in wave number space by using nonlinear spectral analysis (so-called approximated dispersion relation, ADR) [15]. It shows that the genuine nonlinear mechanisms underlying shock capturing schemes have a dramatic impact upon the computed solution, and their actual behavior may be very different from the one predicted on purely linear grounds. The theory can provide useful guidance for the choice of the most cost-effective schemes for specific application, and may constitute a basis for the development of optimized ones. In this paper, we follow this analysis and give the optimized coefficients of the scheme.

In our research, the ADR method of Pirozzoli [15] is simplified. In Pirozzoli's method, a onedimensional linear convection equation is solved numerically and small time-advanced, and then discrete Fourier transform (DFT) is used for the spectral analysis. In our method, only spatial finite difference and DFT operation is used, and time-advancing operation is not needed, so, it is much simpler.

The nonlinear spectral analysis method in this research can be considered as a direct extension of the Fourier analysis method for linear schemes. First, we review the Fourier analysis for a linear scheme.

Consider a linear finite difference scheme defined as

$$
F_{j} \equiv \Delta x f_{j}^{\prime}=\sum_{m=-p}^{q} a_{m} f_{j+m}
$$

Consider the uniform grids $x_{j}=j \Delta x$ and let $f_{j}=e^{i k x_{j}}$, then

$$
F_{j}=\sum_{m=-p}^{q} a_{m} e^{i k\left(x_{j}+m \Delta x\right)}=\hat{F}_{k} e^{i k x_{j}}
$$

where

$$
\hat{F}_{k}=\sum_{m=-p}^{q} a_{m} e^{i m k \Delta x}=\sum_{m=-p}^{q} a_{m} e^{i m \alpha}
$$

and

$$
\alpha \equiv k \Delta x
$$

Define the modified wave numbers

$$
k_{r}=k_{r}(\alpha) \equiv \operatorname{Re}\left(\hat{F}_{k}\right), \quad k_{i}=k_{i}(\alpha) \equiv \operatorname{Im}\left(\hat{F}_{k}\right)
$$

where $k_{r}$ relates to the dissipation error of scheme (2.8) and $k_{i}$ relates to the dispersion error of the scheme.

Consider the exact solution of the difference as

$$
F_{j}^{\text {exact }}=\Delta x\left(\frac{\partial f}{\partial x}\right)_{j}=i k \Delta x e^{i k x_{j}}=i \alpha e^{i k x_{j}}
$$

Compared with eq. (2.9), $k_{r}$ and $k_{i}$ for exact difference (spectral method) are

$$
k_{r}^{\text {exact }}=0, k_{i}^{\text {exact }}=\alpha
$$


Then, we extend this method to nonlinear schemes. Consider a nonlinear scheme defined as

$$
F_{j} \equiv \Delta x f_{j}^{\prime}=F\left(f_{j-p}, \ldots \ldots f_{j+q}\right)
$$

Assume $f_{j}=e^{i k x_{j}}$, which is a sinusoidal function with wave number $k$. Because the scheme (2.14) is nonlinear, $F_{j}$ must contain the harmonic waves of the foundational wave $e^{i k x_{j}}$, that is, $F_{j}=\hat{F}_{k} e^{i k x_{j}}+$ harmonic waves. Where $\hat{F}_{k}$ is the complex amplitude of the foundational wave. When $\alpha \equiv k \Delta x$ is not large, the nonlinear effect of the scheme is not strong, and then the harmonic waves are not as strong as the foundational wave. Then, we have the assumption

$$
F_{j} \approx \hat{F}_{k} e^{i k x_{j}}
$$

The complex amplitude $\hat{F}_{k}$ can be computed by using the DFT

$$
\hat{F}_{k}=\frac{1}{N} \sum_{j=1}^{N} F_{j} e^{-i k x_{j}}
$$

Consider equation (2.15) has the same form as equation (2.9), and we define the modified wave numbers $k_{r}$ and $k_{i}$ as same as that in equation (2.12).

The following gives brief steps of how to use the nonlinear spectral analysis.

1. Select the computational domain $[0,2 \pi]$ and uniform grids $x_{j}=j \Delta x,(j=1,2 \ldots N)(N$ is a large fixed number);

2. Given a fixed wave number $k$ and then obtain values of $f_{j}=e^{i k x_{j}}$ on each grid.

3. Compute the finite difference approximation of $\Delta x\left(\frac{\partial f}{\partial x}\right)_{j}$ by using numerical scheme

$$
F_{j} \equiv \Delta x f_{j}^{\prime}=f_{j+1 / 2}-f_{j-1 / 2}
$$

4. Through the DFT, compute the spectrum with wave number $k$

$$
\hat{F}_{k}=\frac{1}{N} \sum_{j=1}^{N} F_{j} e^{-i k x_{j}}
$$

5. Compute the modified wave numbers

$$
k_{r}=k_{r}(\alpha)=\operatorname{Re}\left(\hat{F}_{k}\right), \quad k_{i}=k_{i}(\alpha)=\operatorname{Im}\left(\hat{F}_{k}\right)
$$

6. Change the value of $k$ and repeat the process of (2)-(5), plot the curves $k_{r}=k_{r}(\alpha)$ and $k_{i}=k_{i}(\alpha)($ where $\alpha \equiv k \Delta x)$.

In the case that the numerical scheme in step (3) is linear, this method gives the same curves of $k_{r}=k_{r}(\alpha)$ and $k_{i}=k_{i}(\alpha)$ as the classical (linear) Fourier analysis [13]. In extended form linear analysis, we call the curve $k_{r}=k_{r}(\alpha)$ as the dissipation curve and call $k_{i}=k_{i}(\alpha)$ as the dispersion curve.

To test this method, we plot the curves of $k_{r}=k_{r}(\alpha)$ and $k_{i}=k_{i}(\alpha)$ for a seventh-order WENO scheme (WENO7) in Figure2 . The values, by using Pirozzoli's ADR method, are also shown in this figure with the definition $k_{r}=-\operatorname{Im}(\Phi(\varphi)), k_{i}=\operatorname{Re}(\Phi(\varphi))$, where $\Phi(\varphi)$ is the modified wave number of ADR method [15]. This figure shows that this method gives the same values as Pirozzoli's ADR method, but this method is much simpler.

2.3.2. Optimization by nonlinear spectral analysis. In Sections 2.1-2.2, a class of sixth-order MP schemes with free parameters (MP-FP6) is given. The free parameters will be specified by using optimization technique through nonlinear spectral analysis addressed in the previous section. After $\xi$ and $\eta$ is specified, the scheme called sixth-order optimized MP scheme (OMP6) is given finally. 

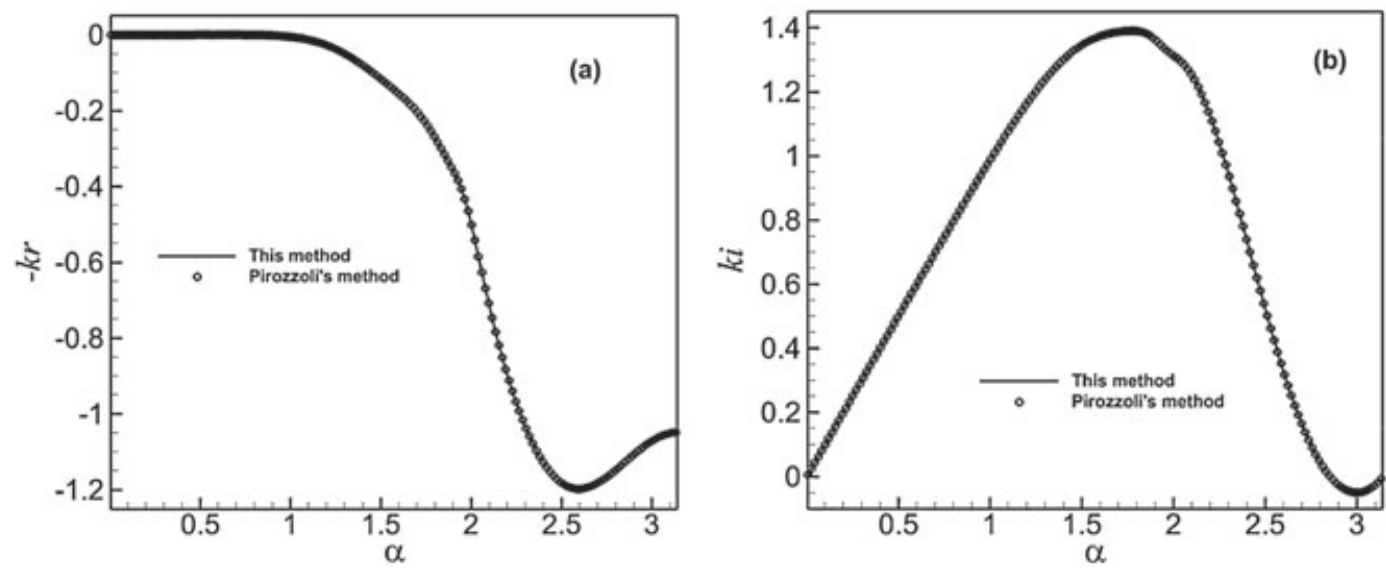

Figure 2. Plot of $k_{r}$ and $k_{i}$ as a function of $\alpha$ for WENO7 scheme, comparison with Pirozzoli's ADR method.
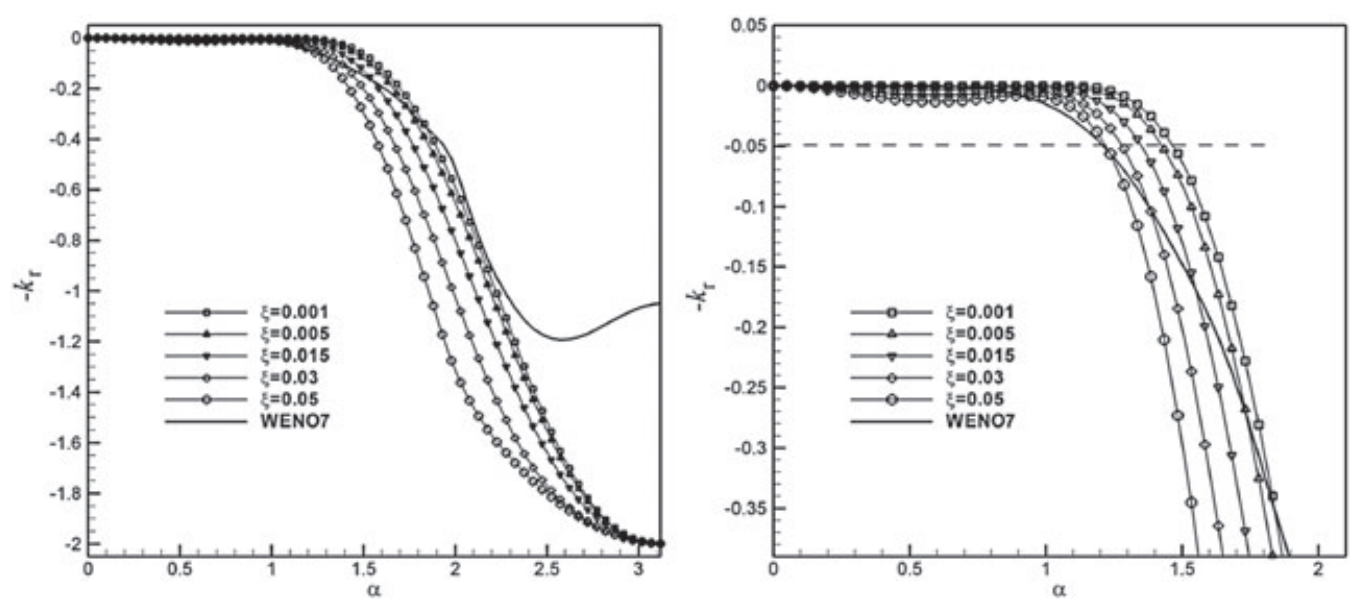

Figure 3. Dissipation curves for monotonicity-preserving-FP6 schemes with different $\xi$ (with $\eta=0$ ).

Equation (2.5) shows that the dissipation of the linear part of MP-FP6 depends only on the parameter $\xi$, and the dispersion depends only on $\eta$. When the nonlinear (limiter) part is adding, MP-FP6's dissipation (or dispersion) depends on both $\xi$ and $\eta$. However, numerical tests show that MP-FP6's dissipation mainly depends on $\xi$, and the effects of $\eta$ on the dissipation are relatively weak, especially in the low wave number range. Similarly, MP-FP6's dispersion mainly depends on $\eta$. To simplify the optimization process, the dissipation and dispersion curves can be optimized independently. We optimize dissipation curve by adjusting $\xi$, and optimize dispersion curve by adjusting $\eta$.

Equation (2.5) requires $\xi \geqslant 0$ because the dissipation (of the linear scheme) must be positive. A scheme with large numerical dissipation is surely not suitable for detailed simulation of complex flows. However, the central scheme without dissipation is insufficient in suppressing the numerical oscillation and leads to instability. Therefore, a small amount of dissipation is needed. In the application to specific physical problem, $\xi$ can be given an appropriate value to avoid over smoothed discontinuities. That is, the optimal dissipation often depends on the problem.

The dissipation curves $k_{r}=k_{r}(\alpha)$ with different $\xi$ of MP-FP6 (sixth-order MP schemes with free parameters) scheme ( $\eta=0$ is specified) by nonlinear spectral analysis are shown in Figure 3, where the dissipation curves of seventh-order Weighted ENO scheme [3] (WENO7) are also shown in this figure. This figure shows that the dissipation becomes larger as the increasing of $\xi$, and this indicates that it is feasible to reduce dissipation by control $\xi$. This figure also shows that the MPFP6 schemes with $\xi \leqslant 0.05$ have higher wave number resolution range than WENO7 as we use 
the criterion $k_{r} \leqslant 0.05$. This also means that the MP-FP6 schemes with $\xi \leqslant 0.05$ are less dissipative than WENO7. Numerical tests show that MP-FP6 schemes with $\xi=0.001$ are robust enough for supersonic/hypersonic flows. We suggest that $\xi=0.001$. In the extreme case such as strong shock/turbulence interaction, users can choose larger $\xi$ (such as $\xi=0.015$ or $\xi=0.02$ ) for high robustness.

After $\xi$ is determined by the dissipation optimization, we specify another free parameter $\eta$ by the optimization of dispersion. Define

$$
G(\alpha) \equiv \bar{\alpha} \Delta \alpha \equiv \bar{\alpha}\left[\alpha-k_{i}(\alpha)\right], \quad(\bar{\alpha}=\pi / 2) .
$$

Because the ideal dispersion curve is $k_{i}=\alpha$ (which means no dispersion error), higher $|G(\alpha)|$ represents larger dispersion error. Here, we require

$$
G(\alpha) \geqslant 0,
$$

which means that $k_{i}(\alpha) \leqslant \alpha$, that is, the MP-FP6 scheme should not be a 'fast scheme' (the propagation speed of numerical waves is faster than that of the physical waves). Curves of $k_{i}(\alpha)$ and $G(\alpha)$ for MP-FP6 schemes with $\eta=-1.3 / 140 \sim 0.8 / 140$ (specify $\xi=0.001$ ) are shown in Figures 4-5. We define the maximum wave number range of dispersion resolution as

$$
\alpha_{D}=\max (\alpha): G(\alpha) \leqslant 0.02 .
$$
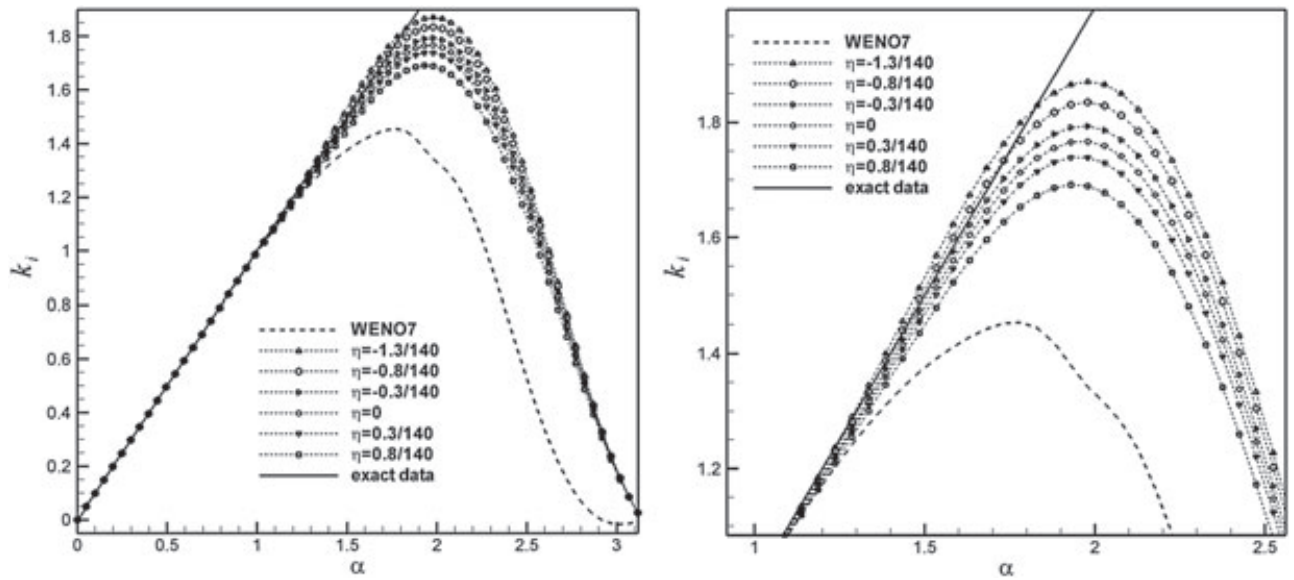

Figure 4. Dispersion curves for different $\eta$.
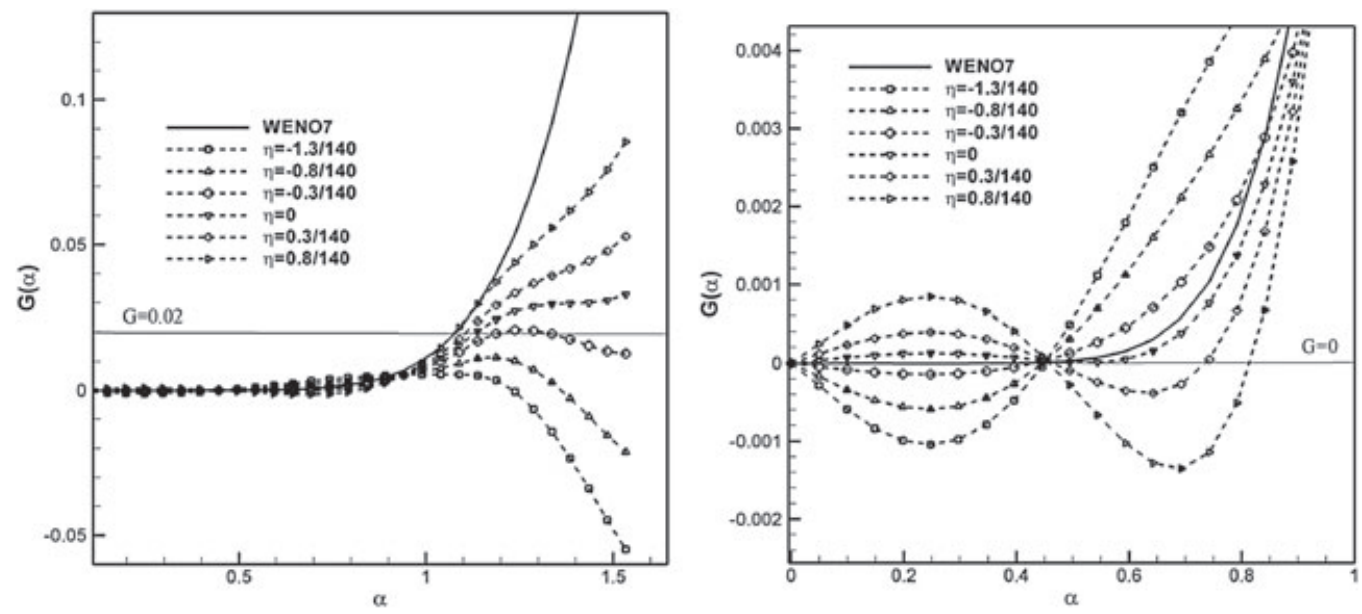

Figure 5. $G(\alpha)$ curves for different $\eta$. 
Parameter $\eta$ is then determined by the criteria of maximized $\alpha_{D}$ and $G(\alpha) \geqslant 0$. Finally, we suggest $\eta=0$ as the optimized value.

By specifying the free parameters $\xi=0.001$ and $\eta=0$, the final scheme $((2.4),(2.6),(2.7))$ is then given, and we call it optimized sixth-order MP scheme (OMP6).

The dispersion curves of WENO7 scheme are also shown in Figures 3-4, which show that OMP6 scheme has wider wave number resolution range than the WENO7 scheme.

Figure 6 gives the dispersion curve of linear scheme (2.4) by Fourier analysis. Comparison with Figure 4 shows that the nonlinear (limiter) part changes the dispersion property of the scheme obviously. For example, linear scheme (2.4) with $\eta=-1.3 / 140$ is 'slow scheme' $\left(k_{i}<\alpha\right)$, but the MP-FP6 scheme ((2.4) with MP limiter) with $\eta=-1.3 / 140$ becomes 'fast scheme' $\left(k_{i}>\alpha\right)$ in the low wave number range. This indicates that the optimization based on the linear analysis cannot give the optimal solution.

Finally, the flux of OMP6 schemes for conservation law (2.1) with $f=a u, a>0$ is

$$
f_{j+1 / 2}=\left\{\begin{array}{lrr}
f_{j+1 / 2}^{\text {Linear }} \quad \text { if } \quad\left(f_{j+1 / 2}^{\text {Linear }}-f_{j}\right)\left(f_{j+1 / 2}^{\text {Linear }}-f_{j+1 / 2}^{\mathrm{MP}}\right) \leqslant \varepsilon & \left(\varepsilon=10^{-10}\right) \\
f_{j+1 / 2}^{\text {Linear }}+\min \bmod \left(f_{j+1 / 2}^{\min }-f_{j+1 / 2}^{\text {Linear }}, f_{j+1 / 2}^{\max }-f_{j+1 / 2}^{\text {Linear }}\right) & \text { otherwise }
\end{array}\right.
$$

where

$$
\begin{aligned}
f_{j+1 / 2}^{\text {Linear }}= & \frac{3}{6000} f_{j+4}+\frac{79}{6000} f_{j+3}-\frac{737}{6000} f_{j+2}+\frac{3595}{6000} f_{j+1} \\
& +\frac{3805}{6000} f_{j}-\frac{863}{6000} f_{j-1}+\frac{121}{6000} f_{j-2}-\frac{3}{6000} f_{j-3}
\end{aligned}
$$

$f_{j+1 / 2}^{\mathrm{MP}} f_{j+1 / 2}^{\max }$ and $f_{j+1 / 2}^{\min }$ are defined in (2.7).

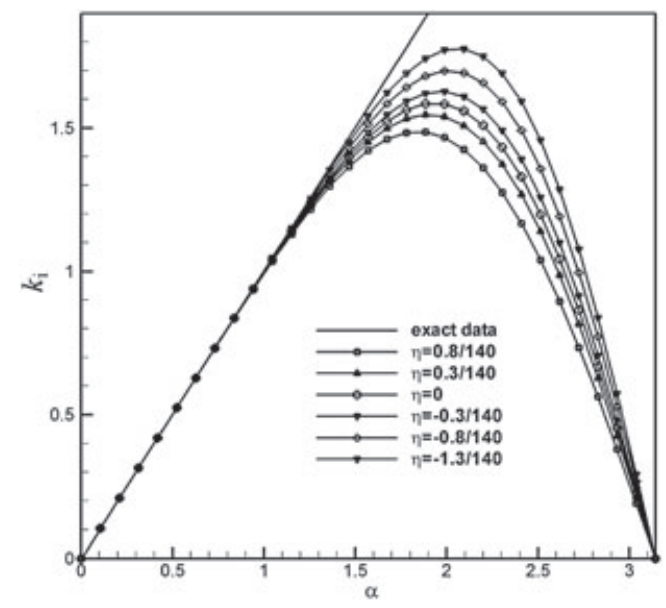

Figure 6. Dispersion curves for linear schemes (2.4) with different $\eta$.

Table I. $L_{1}$ and $L_{\infty}$ errors and order with $u(x, 0)=\sin (\pi x)$.

\begin{tabular}{lccccc}
\hline Method & Numbers of zone & $L_{1}$ & $L_{1}$ & $L_{\infty}$ & $L_{\infty}$ \\
\hline \multirow{4}{*}{ WENO7 } & error & order & error & order & \\
$\varepsilon=10^{-40}$ & 10 & $5.37 \mathrm{E}-03$ & - & $5.97 \mathrm{E}-03$ & - \\
& 20 & $8.56 \mathrm{E}-05$ & 5.97 & $1.21 \mathrm{E}-04$ & 5.63 \\
& 40 & $1.24 \mathrm{E}-06$ & 6.11 & $2.94 \mathrm{E}-06$ & 5.35 \\
& 80 & $1.91 \mathrm{E}-08$ & 6.02 & $8.14 \mathrm{E}-08$ & 5.18 \\
OMP6 & 160 & $2.92 \mathrm{E}-10$ & 6.03 & $2.30 \mathrm{E}-09$ & 5.15 \\
& 10 & $3.62 \mathrm{E}-04$ & - & $2.42 \mathrm{E}-04$ & - \\
& 20 & $7.84 \mathrm{E}-06$ & 5.53 & $5.75 \mathrm{E}-06$ & 5.40 \\
& 40 & $1.30 \mathrm{E}-07$ & 5.92 & $9.83 \mathrm{E}-08$ & 5.87 \\
& 80 & $2.04 \mathrm{E}-09$ & 5.99 & $1.57 \mathrm{E}-09$ & 5.97 \\
& 160 & $3.18 \mathrm{E}-11$ & 6.00 & $2.48 \mathrm{E}-11$ & 5.98 \\
\hline
\end{tabular}


As for the conservation law (2.1) with $f=a u, a<0$, the numerical flux can be constructed similarly. In fact, the expression of numerical flux for (2.1) with $f=a u, a<0$ is easy to obtain by simply replacing the subscript ' $j+k$ ' with ' $j-k$ ' in (2.7), (2.17), and (2.18) (such as replace $j+2$ with $j-2$, replace $j-1$ with $j+1$, and replace $j+1 / 2$ with $j-1 / 2$ ).

In the extreme case such as very strong shock, the users can choose larger $\xi$ (such as 0.015 or $0.02)$ in (2.4) for higher robustness.

\section{EXTENSION TO THE EULER EQUATION}

In this section, the OMP6 scheme is extended to the Euler equations. The one-dimensional Euler equations can be written as the following conservative form

$$
\frac{\partial \mathbf{U}}{\partial t}+\frac{\partial \mathbf{F}(\mathbf{U})}{\partial x}=0
$$

where $\mathbf{U}=(\rho, \rho u, E)^{\mathrm{T}}$ is the vector of conservative variables and $\mathbf{F}(\mathbf{U})=\left(\rho u, \rho u^{2}+p, u(E+p)\right)^{\mathrm{T}}$ is the vector of flux. The semi-discrete conservative finite difference scheme can be written as

$$
\frac{\partial \mathbf{U}}{\partial t}+\frac{1}{h}\left(\mathbf{F}_{j+1 / 2}-\mathbf{F}_{j-1 / 2}\right)=0
$$

where $\mathbf{F}_{j+1 / 2}$ is the inviscid numerical fluxes, which are computed by the characteristic decomposition. This consists of the following steps:
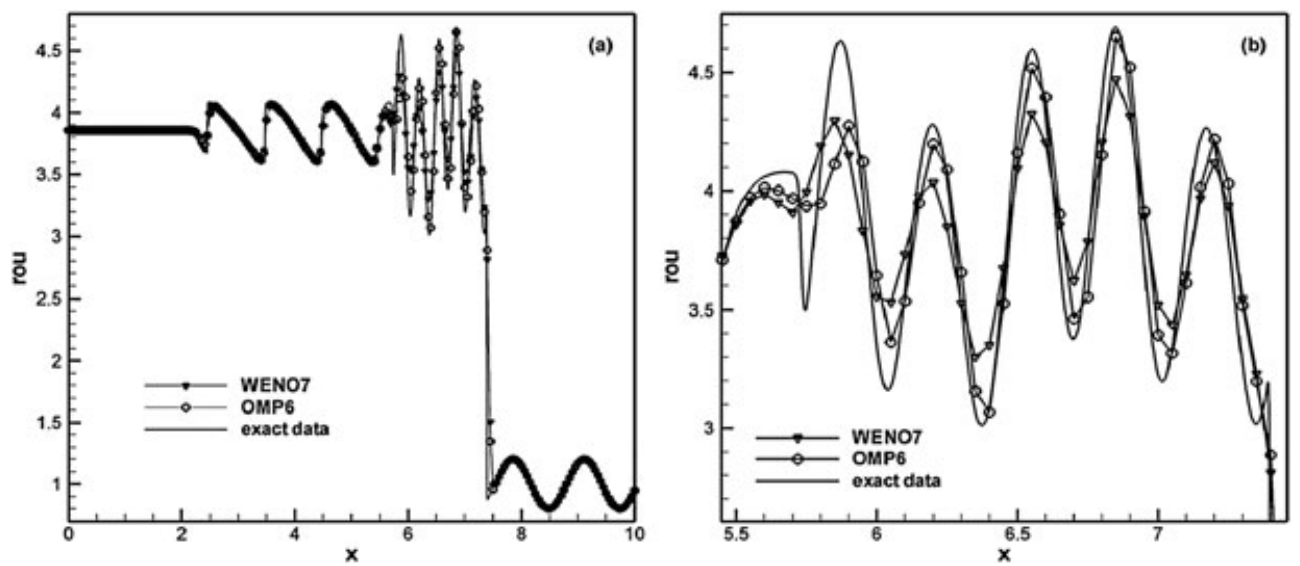

Figure 7. Shu-Osher problem. Distribution of density at $t=1.8$ for WENO7 and OMP6, 200 grid points.
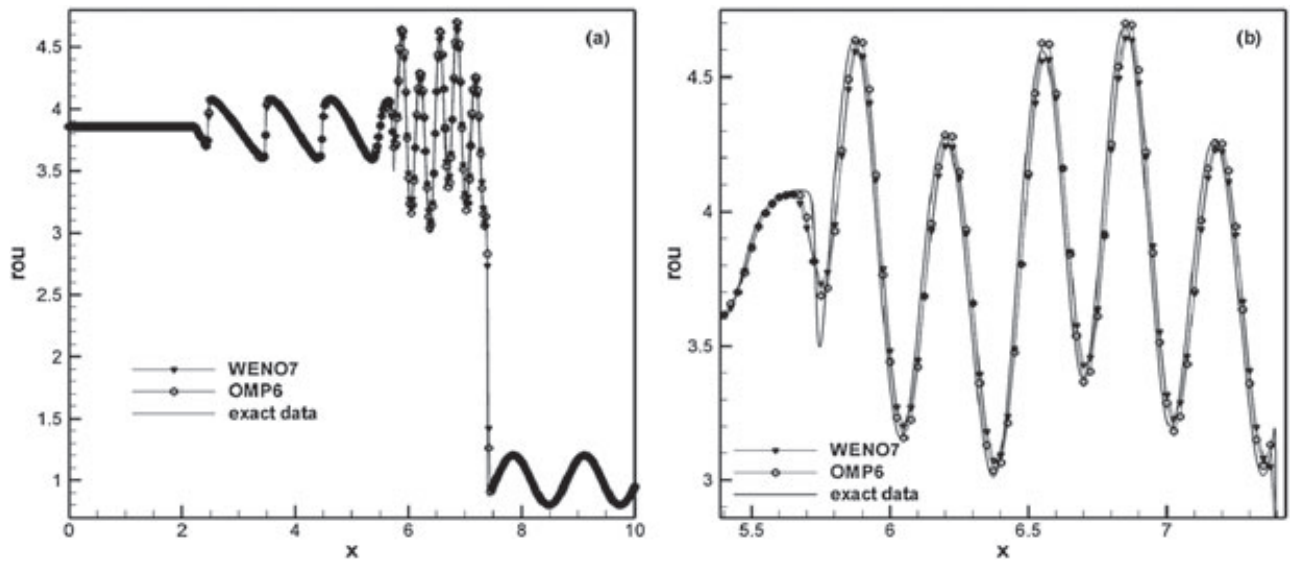

Figure 8. Shu-Osher problems. Distribution of density at $t=1.8$ for WENO7 and OMP6, 400 grid points. 
1. Use the flux vector splitting methods (such as Lax-Friedrichs splitting [3], Steger-Warming splitting [16], or Van Leer splitting [17] to split the fluxes into a positive part and a negative part, that is, $\mathbf{F}=\mathbf{F}^{+}+\mathbf{F}^{-}$;

2. At each fixed $x_{j+1 / 2}$, the average state $\mathbf{U}_{j+1 / 2}$ is computed by the arithmetic average or the Roe [18] average;

3. The eigenvalues $\lambda_{j+1 / 2}^{i}(i=1,2,3)$, the left eigenvectors $\mathbf{l}_{j+1 / 2}^{i}(i=1,2,3)$ and the right eigenvectors $\mathbf{r}_{j+1 / 2}^{i}(i=1,2,3)$ are computed in terms of $\mathbf{U}_{j+1 / 2}$;

4. The local characteristic decompositions of the flux functions at the stencils $x_{m}$ are computed by

$$
\begin{aligned}
\mathbf{w}_{m}^{+(i)} & =\mathbf{l}_{j+1 / 2}^{i} \bullet \mathbf{F}_{m}^{+},(i=1,2,3 ; m=j-3, \ldots, j+4) \mathbf{w}_{m}^{-(i)} \\
& =\mathbf{r}_{j+1 / 2}^{i} \bullet \mathbf{F}_{m}^{-},(i=1,2,3 ; m=j-3, \ldots, j+4)
\end{aligned}
$$

5. Compute the numerical flux $\mathbf{w}_{j+1 / 2}^{+(i)}$ and $\mathbf{w}_{j+1 / 2}^{-(i)}$ by using OMP6.

6. Transform back into physical space to obtain flux $\mathbf{F}_{j+1 / 2}=\mathbf{F}_{j+1 / 2}^{+}+\mathbf{F}_{j+1 / 2}^{-}$

\section{NUMERICAL TESTS}

In the following tests, the seventh-order WENO scheme (WENO7) [3], seventh-order MP scheme (MP7) [5], and the current OMP6 scheme are used to compute inviscid numerical flux. The Lax-Friedrichs flux vector splitting method is used to split the fluxes into a positive and a negative part for one-dimensional and two-dimensional tests and for three-dimensional tests is the Steger-Warming splitting method. For viscous case, eighth-order central scheme is used to discrete viscous terms. The third-order TVD type Runge-Kutta method [3] is used for time advance. All test cases except the three-dimensional direct numerical simulation (DNS) are run on a PC with a Core i $7 \mathrm{CPU}$ at $2.67 \mathrm{GHz}$ (run as single-threaded serially).

Table II. The CPU times needed for the Shu-Osher problem (18 000 time steps).

\begin{tabular}{lcc}
\hline Method & \multicolumn{2}{c}{ Total CPU time (s) } \\
\hline & 200 points & 400 points \\
WENO7 & 13.39 & 26.67 \\
MP7 & 9.01 & 13.12 \\
OMP6 & 9.03 & 13.20 \\
\hline
\end{tabular}
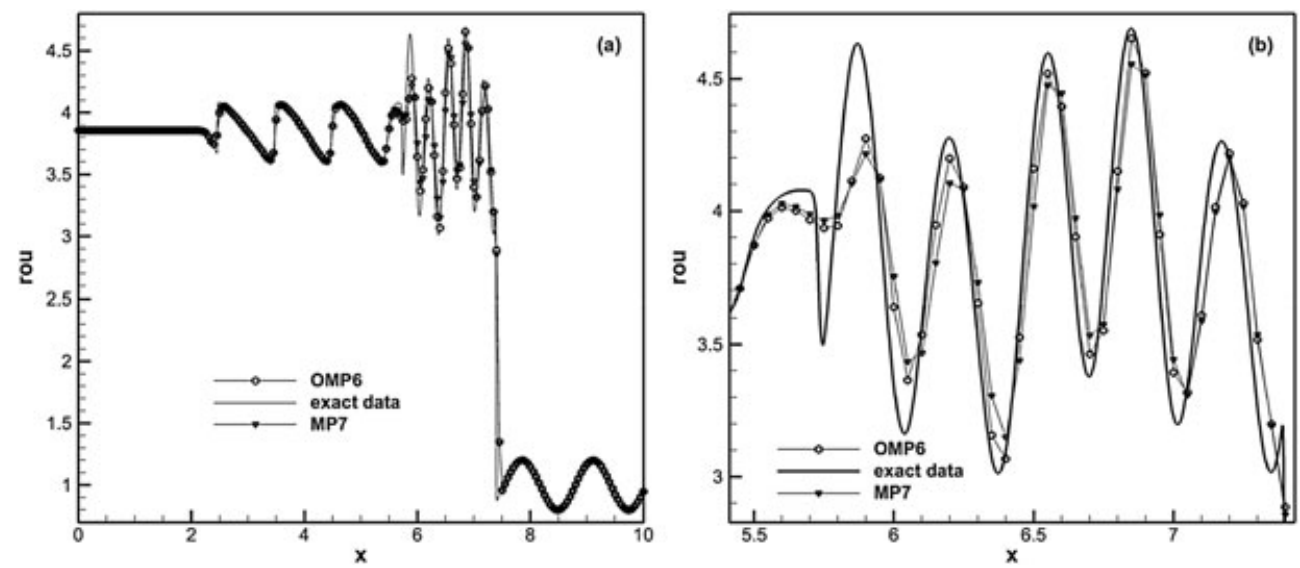

Figure 9. Shu-Osher problems. Distribution of density at $t=1.8$ for MP7 (upwind7+MP) and OMP6, 200 points. 


\subsection{One-dimensional problems}

\subsubsection{Convergence study for the advection equations. [3, 19]}

The following equation is solved on the domain $[-1,1]$ with periodic boundary conditions.

$$
\left\{\begin{array}{l}
u_{t}+u_{x}=0 \\
u(x, 0)=\sin (\pi x)
\end{array}\right.
$$

The computed $L_{1}$ and $L_{\infty}$ errors and order of accuracy are listed in Table I. The errors were measured at $t=1$, and the time step has been adjusted to $\Delta t=(\Delta x)^{3}$. The results in Table I tell us that the OMP6 scheme gives the theoretical order of sixth-order accuracy starting at about 80 grid points. And, the errors of the OMP6 are significantly less than the WENO7 schemes no matter on the coarse or the fine grid.

\subsubsection{Shu-Osher problem. [20]}

This test indicates that Mach 3 shock interacts with a density disturbance. And, this is a good model to test the schemes resolution for both shock waves and small-scale waves. The governing equations are one-dimensional Euler equations and solved on the spatial domain $x \in[0,10]$. The initial conditions are

$$
\left\{\begin{array}{l}
\rho=3.857143, u=2.629369, p=10.333333 \text { when } \mathrm{x}<1 \\
\rho=1+0.2 \sin (5 x), u=0, \quad p=1 \quad \text { when } \mathrm{x} \geqslant 1
\end{array} .\right.
$$

The solution is advanced up to $t=1.8$ with 200 and 400 points. Because the real exact solution is unknown, the 'exact' solution here is obtained numerically by 4000 points. Figure 7 presents
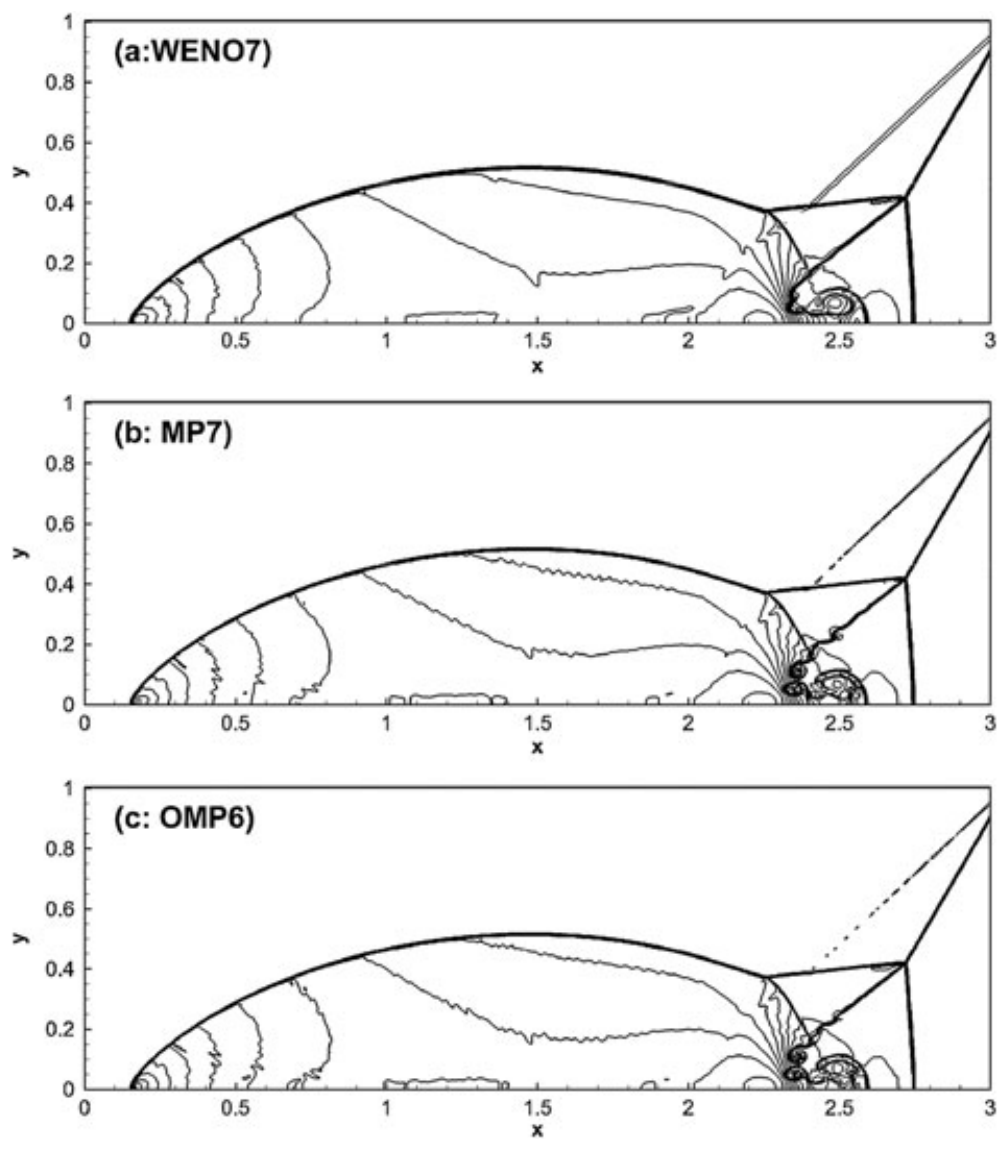

Figure 10. Double-Mach reflection of a Mach 10 shock wave, density profile at $t=0.2,960 \times 240$ grid: (a) WENO7, (b) MP7, and (c) OMP6 (from 1.731 to 20.92 with 30 equally spaced contours). 
the comparison between the WENO7 and the OMP6 scheme for 200 grid points. Figure $7 \mathrm{~b}$ is the locally enlarged plot of Figure 7a. Clearly shown in Figure 7, the OMP6 gives better resolution than the classical WENO7 scheme, especially, in the region of high wave number behind the shock. The improved dispersion property is responsible for its better high wave number simulation capability. But, for the first wave behind the shock waves, the result is not as good as the others. The reason is that the first wave contains only four grid points, yet other waves contains six grid points. It has been beyond the range of optimal wave number. The improved dissipation property is responsible for its better shock capturing capability. The dissipation should be reduced as much as possible; but on the other hand, it should suppress the numerical oscillation. In Figure 8, the results are compared between the OMP6 and WENO7 using 400 grid points. One can conclude that both schemes give a better resolved density waves on this mesh, but at a lower cost for the OMP6 scheme which is about half of computational time less expensive than the WENO7 as shown in Table II.

Figure 9 shows the comparison between the OMP6 scheme and the original seventh-order MP scheme (seventh-order upwind scheme with MP limiter, marked as MP7), which is given in ref. [5] on 200 grid points. The figure shows that the optimization of dispersion and dissipation obtains better resolution.

\subsection{Two-dimensional problems}

\subsubsection{Double Mach reflection problem. [21]}

The governing equations are two-dimensional Euler equations, and the computational domain for this problem is chosen to be $[0,4] \times[0,1]$. The reflecting wall lies at the bottom of the computational
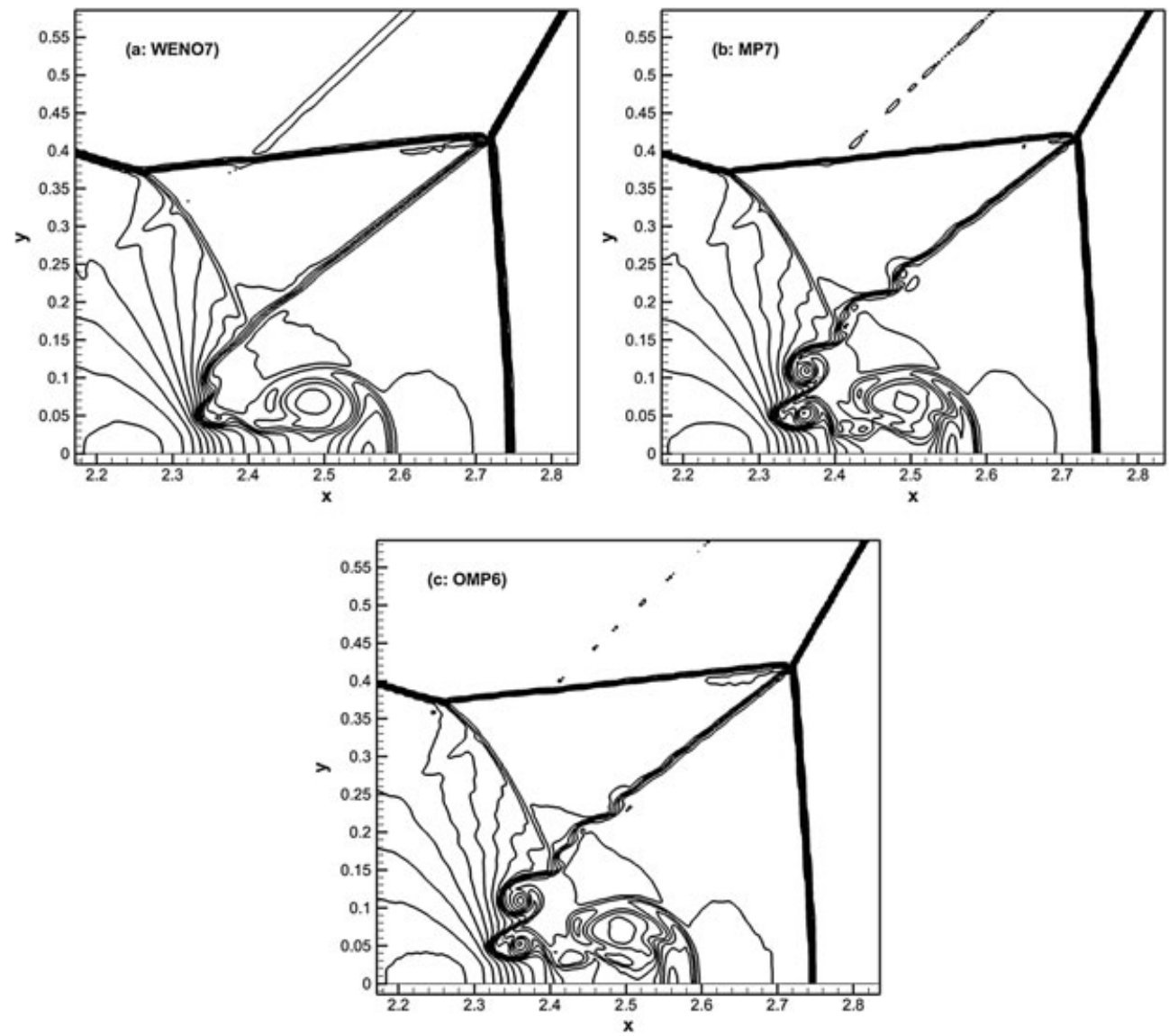

Figure 11. Close-up view of the 'blow-up' region of Figure 11: (a) WENO7, (b) MP7, and (c) OMP6 (from 1.731 to 20.92 with 30 equally spaced contours). 
domain starting from $x=1 / 6$. Initially, a right-moving Mach 10 shock wave in air $(\gamma=1.4)$ is positioned at $x=1 / 6, y=0$, and makes a $60^{\circ}$-angle with the $x$-axis. For the bottom boundary, the region from $x=0$ to $x=1 / 6$ is always assigned the initial values. The boundary with $x>1 / 6$ on the $x$-axis is taken to be a reflecting boundary. At the top boundary of our computational domain, the flow values are set to describe the exact motion of the Mach 10 shock wave. It is a test case involving both strong shock and multiple stems. The results are shown at a simulation time of 0.2 , and the grid resolutions are $960 \times 240$ and $1920 \times 480$ points.

The density contours obtained by the WENO7, MP7, and OMP6 scheme are presented in Figures 10-13, and the total CPU times for per 100 steps are shown in Table III. It can be seen that both schemes capture the features of the solution such as the Mach stem and the wall jet. All the figures show that the OMP6 scheme resolves better the wave structure near the second triple point and predicts a stronger jet near the wall. And, as shown in Figures 11 and 13, the OMP6 scheme achieves higher resolution especially in the region near the Mach stems where the OMP6 scheme can capture the rollup of the slip line more clearly and is less dissipative at small scales (marked by the arrows in the figure).
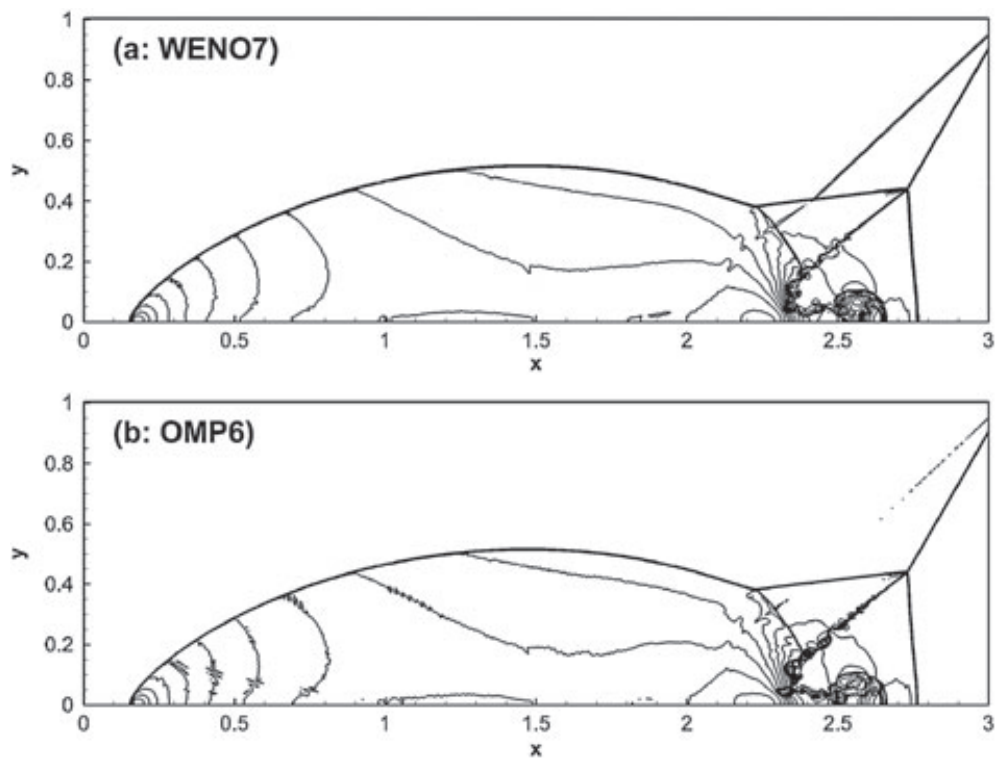

Figure 12. Double Mach reflection of a Mach 10 shock wave, density profile at $t=0.2,1920 \times 480$ grid: (a) WENO7 and (b) OMP6 (from 1.731 to 20.92 with 30 equally spaced contours).
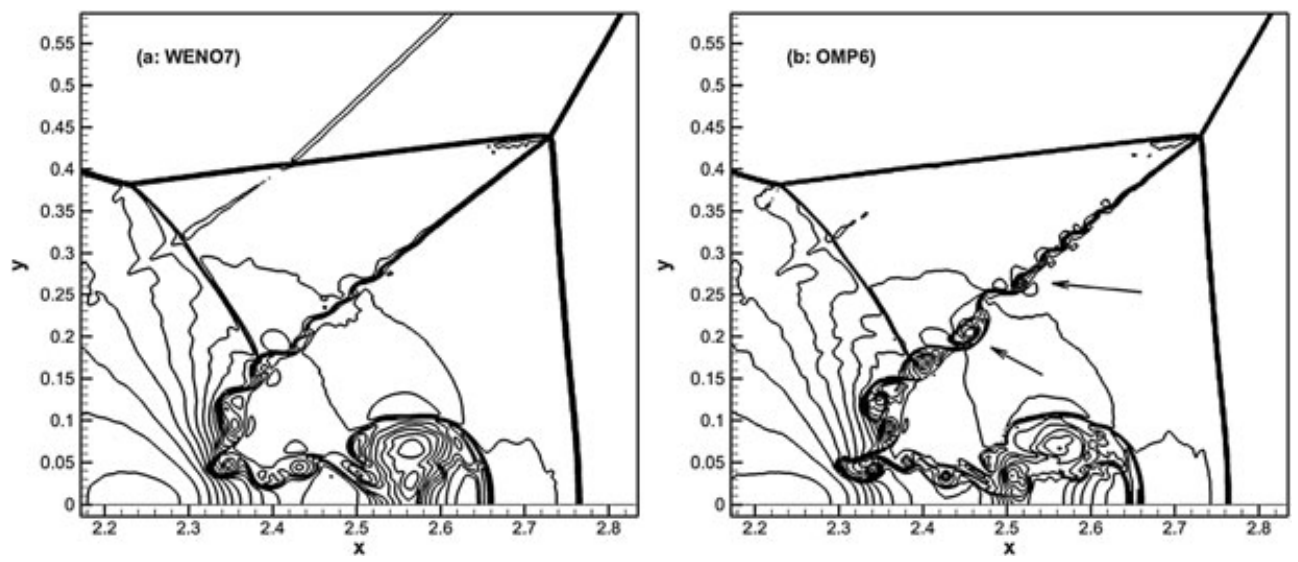

Figure 13. Close-up view of the 'blow-up' region of Figure 13: (a) WENO7 and (b) OMP6 (from 1.731 to 20.92 with 30 equally spaced contours). 
Table III. The total CPU times needed for the Double Mach reflection problem (100 time steps).

\begin{tabular}{lcccccc}
\hline Mesh & \multicolumn{3}{c}{$960 \times 240$} & \multicolumn{3}{c}{$1920 \times 480$} \\
\hline Method & WENO7 & MP7 & OMP6 & WENO7 & MP7 & OMP6 \\
CPU time (s) & 350.3 & 211.2 & 212.7 & 1423.3 & overflow & 850.3 \\
\hline
\end{tabular}

Comparing Figure 11c with the results in [22] (Figure 1), the results obtained by the OMP6 on a $960 \times 240$ grid are similar to the classical WENO9 on a $1920 \times 480$ grid. Figure 12 shows the density contours obtained by the OMP6 and the WENO7 on a $1920 \times 480$ grid. The contours zoomed around the contact discontinuity and Mach stem region are given in Figure 13. In the close-up view of the 'blow-up' region, these results show better resolution in comparison with the result obtained by the classical WENO9 on a $3840 \times 960$ grid in [22].

This test shows that the OMP6 scheme has a higher resolution of small-scale waves for less dissipation errors than the classical WENO7 and have the same capability to capture strong shock waves as the WENO schemes. CPU times in Table III shows that the computing efficiency of OMP6 is very close to that of MP7 (difference is less than 1\%), and the efficiency of both OMP6 and MP7 are much higher than that of WENO7.

The computing with MP7 on $1290 \times 480$ grid overflows at $t=0.134$ (negative pressure occurs in the computation domain); however, the computing with OMP6 does not overflow in the same grid, and this test case shows that the OMP6 is more robust than MP7.

4.2.2. Rayleigh-Taylor instability. [22] Rayleigh-Taylor instability happens on an interface between fluids with different densities when acceleration is directed from the heavy fluid to the light fluid. The instability has a fingering nature, with bubbles of light fluid rising into the ambient heavy fluid and spikes of heavy fluid falling into the light fluid. Previous studies show that there are many small structures. It is a good example to test the resolution of the numerical schemes.

The problem is set up as follows: the computational domain is $\left[0, \frac{1}{4}\right] \times[0,1]$; initially, the interface is at $y=1 / 2$, the heavy fluid with $\rho=2$ is below in the interface and the light fluid with $\rho=1$ is above the interface with the acceleration in the positive $y$-direction; the pressure $p$ is continuous across the interface; a small perturbation is given to the velocity component in $y$ direction. So, for $0 \leqslant y \leqslant 1 / 2, \rho=2, u=0, p=2 y+1, v=-0.025 c \cdot \cos (8 \pi x)$, and for $1 / 2 \leqslant y \leqslant 1, \rho=1, u=0, p=y+\frac{3}{2}, v=-0.025 c \cdot \cos (8 \pi x)$, where $c$ is the sound speed $c=\sqrt{\frac{\gamma p}{\rho}}$, and the ratio of specific heats $\gamma=\frac{5}{3}$. A source term $\rho$ is added to the right-hand side of the third equation, and $\rho v$ is added to the fourth equation of the Euler system. The final simulation time is $t=1.95$.

For comparing the numerical results, the mesh refinement study is used with three uniform meshes: $h=1 / 240,1 / 480,1 / 960$. Figure 14 shows the density contours with 15 equally spaced contour lines from $\rho=0.952269$ to $\rho=2.14589$ obtained by the WENO7, MP7, and OMP6 schemes for $h=1 / 240,1 / 480$. The total CPU times are shown in Table IV. Figure 14 shows that the OMP6 scheme resolves finer results in comparison with WENO7 and MP7 schemes (see the region marked by the arrows in Figure 14). It is obvious that the OMP6 scheme is much less dissipative at small scales and produces much more small vortices in the shear layer than the WENO7 and MP7 schemes. This indicates that OMP6 scheme has better resolution in complicated solution structures than WENO7 and MP7 schemes.

Figure 15 shows the density contours with 15 equally spaced contour lines from $\rho=0.952269$ to $\rho=2.14589$ obtained by the WENO7, MP7, and OMP6 schemes for $h=1 / 960$. Obviously, again, the OMP6 scheme resolves considerably finer results in comparison with the two other schemes.

Comparing Figures 14 and 15 again with the results in [22] (Figure 2), the results obtained by the OMP6 with $h=1 / 240$ are similar to the classical WENO9 with $h=1 / 480$. The OMP6 with $h=1 / 480$ has qualitatively the same resolution as WENO9 with $h=1 / 960$, and the same for the OMP6 with $h=1 / 960$ and the WENO9 with $h=1 / 1920$. 

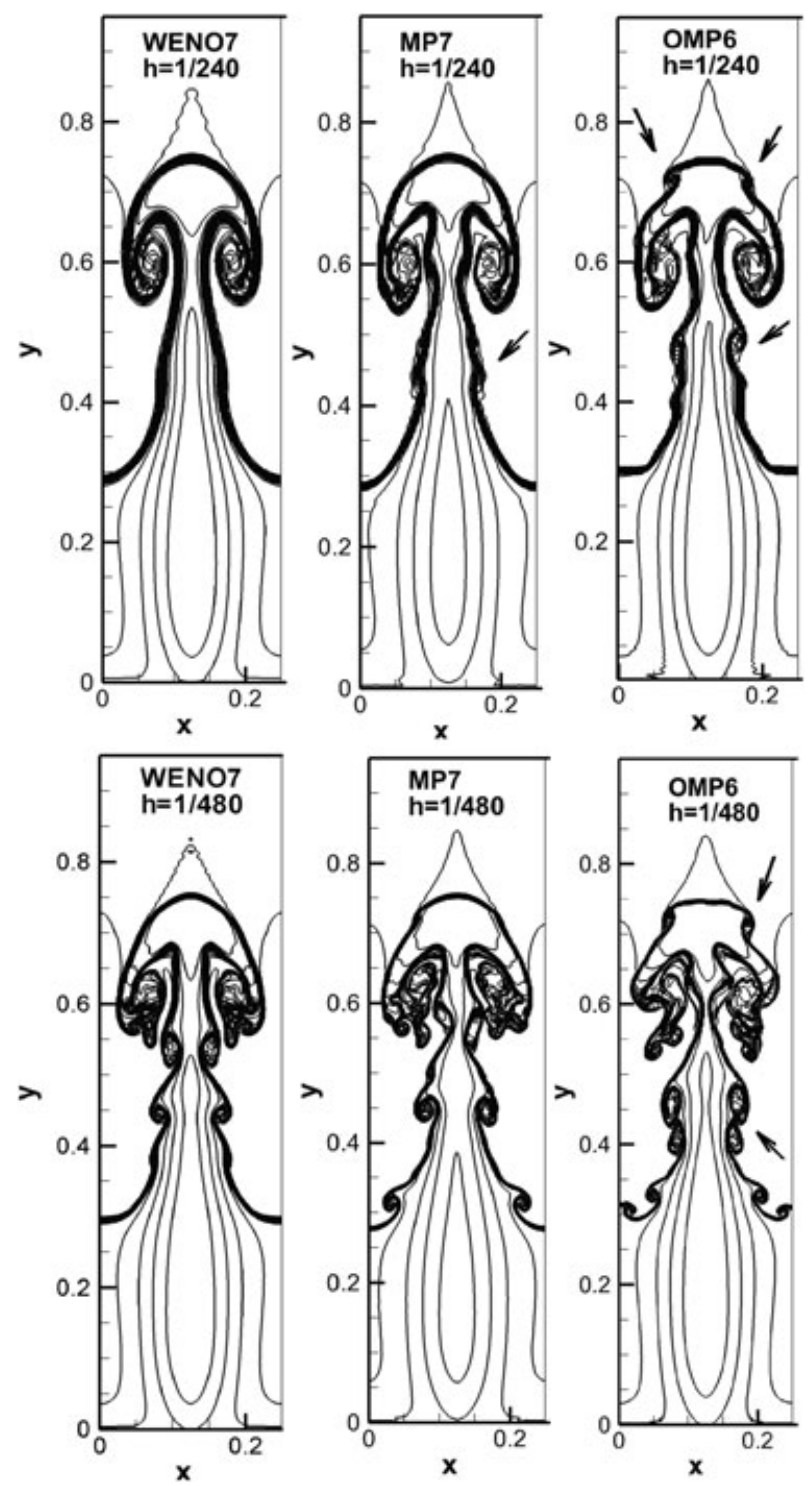

Figure 14. Rayleigh-Taylor instability: density profile at $t=1.95$, by WENO7, MP7, and OMP6 scheme with $h=1 / 240,1 / 480$, (from 0.952269 to 2.14589 with 15 equally spaced contours).

Table IV. The total CPU times needed for the Rayleigh-Taylor instability (100 time steps).

\begin{tabular}{lccccccccc}
\hline Mesh & \multicolumn{3}{r}{$h=1 / 240$} & \multicolumn{3}{r}{$h=1 / 480$} & \multicolumn{3}{c}{$h=1 / 960$} \\
\hline Method & WENO7 & MP7 & OMP6 & WENO7 & MP7 & OMP6 & WENO7 & MP7 & OMP6 \\
CPU time (s) & 354.2 & 205.2 & 208.5 & 1423.5 & 845.1 & 849.2 & 5695.1 & 3420.1 & 3423.2 \\
\hline
\end{tabular}

The previous two tests contain both discontinuities and complex solution structure, which are of critical importance and difficult for numerical simulation. Through analysis, it can be said that the OMP6 scheme with half of mesh points in each direction can achieve the numerical resolution by using the WENO9 scheme. Moreover, the OMP6 has much higher computational efficiency than the WENO7 as shown in Tables IV.

\subsection{Direct numerical simulation of decaying compressible isotropic turbulence [23]}

Direct numerical simulation of compressible isotropic turbulence [23] is a good case to test the resolution of small-scale fluctuations. The computational domain is $[0,2 \pi] \times[0,2 \pi] \times[0,2 \pi]$, and the 

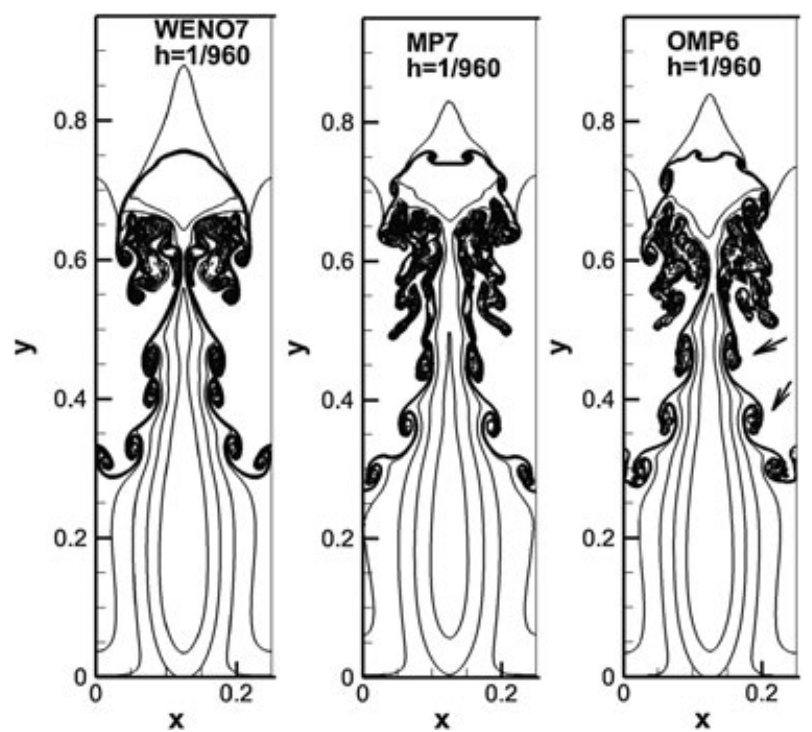

Figure 15. Rayleigh-Taylor instability: density profile at $t=1.95$, by WENO7, MP7, and OMP6 scheme with $h=1 / 960$, (from 0.952269 to 2.14589 with 15 equally spaced contours).

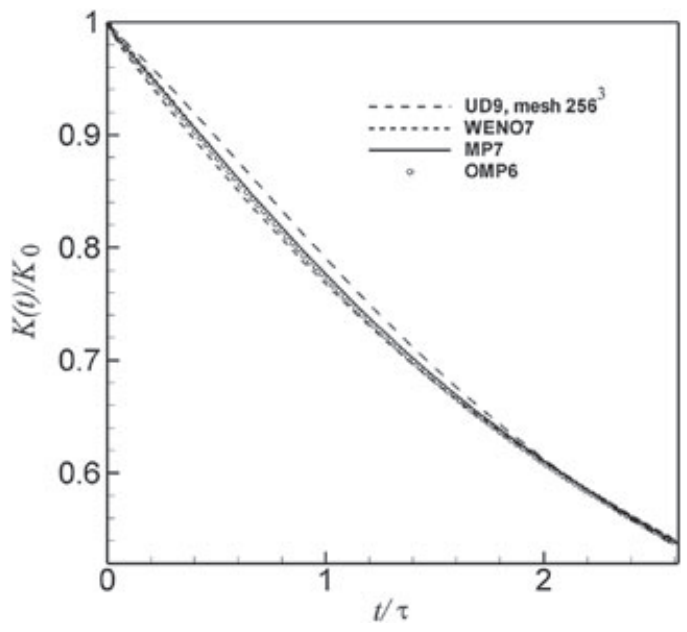

Figure 16. Temporal evolution of turbulent kinetic energy.

mesh contains $128^{3}$ uniform points. The boundary conditions are periodic in all three directions. In this study, we choose the case with initial turbulent Mach number $M_{t 0}=0.2$ and initial Taylor Reynolds number $R e_{\lambda}=97$. During the development of the turbulence, numerical dissipation will speed up the time decay process. So, we focus on the decay process of the turbulent energy and the spectrum for kinetic energy.

Figure 16 shows the temporal evolution of turbulent kinetic energy $K(t)=\frac{1}{2}\left\langle\rho u_{i} u_{i}\right\rangle$ for the WENO7, MP7, and OMP6 schemes on meshes with $128^{3}$ grid points, where $\tau$ is the large eddy turnover time at initial time [23]. For comparison, we conduct another high resolution case with $256^{3}$ uniform points and higher older scheme (9th upwind scheme for inviscid term and 10th central term for viscous term), and the result can be seen as 'exact solution.' The Comparison with the 'exact solution' shows that the decaying curve of WENO7 is faster than that of OMP6 and MP7, which shows that OMP6 and MP7 are less dissipative than WENO7.

Figure 17 shows the kinetic energy spectral [23] at $t=1.5(t / \tau=0.99)$ for the WENO7, MP7, OMP6, and the 'exact solution.' This figure shows that, compared with the spectrum computed by 


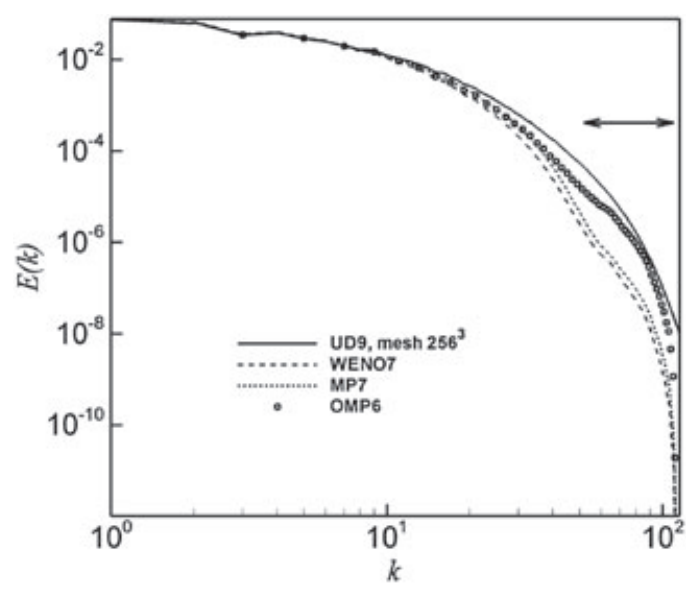

Figure 17. Kinetic energy spectral at $t=1.5(t / \tau=0.99)$.

WENO7and MP7, the spectrum computed by OMP6 is closer to the 'exact solution,' especially in the high wave number range (marked by the arrow in Figure 17). This figure shows that OMP6 has much higher resolution for small scales than that of the WENO7 and MP7 schemes.

\section{CONCLUDING REMARKS}

In this paper, sixth-order MP optimized schemes (OMP6) is developed on the basis of the optimization and MP technique. The nonlinear spectral analysis is used for the purpose of minimizing the dispersion errors and controlling the dissipation errors. The new scheme (OMP6) is simple in expression and is easy to be used in CFD codes. We test the OMP6 schemes through the Shu-Osher problem, double Mach reflection problem, Rayleigh-Taylor instability problem, and the DNS of decaying compressible turbulence. Though these test cases, we compared OMP6 scheme with seventh-order WENO scheme (WENO7) and original seventh-order MP scheme (MP7). All numerical tests show that the OMP6 scheme has robust shock capturing capability and high resolution for the small-scale waves due to fewer numerical dispersion and dissipation errors. Moreover, the new scheme has higher computational efficiency than the well-used WENO schemes.

\section{ACKNOWLEDGEMENTS}

Thanks to Profs Dexun Fu and Yanwen Ma in the Institute of Mechanics, CAS for the advices of numerical methods. Thanks to Prof Yuxin Ren in Tsinghua University for the helpful discussion on the optimization method. This work was supported by the National Natural Science Foundation of China (Nos. 10632050, 10872205, 11072248), the 973 project (Grant Nos. 2009CB724100), the 863 program (No. 2012AA01A304), and project of CAS INFO-115-B0. The authors thank National Supercomputing Center in Tianjin (NSCC-TJ), Supercomputing Center of Chinese Academy of Sciences (SCCAS) and Shanghai Supercomputer Center (SSC) for providing computer time.

\section{REFERENCES}

1. Moin P, Mahesh K. Direct numerical simulation: a tool in turbulence research. Annual Fluid Mechanics 1998; 30:539-578.

2. Liu XD, Osher S, Chan T. Weighted essentially non-oscillatory schemes. Journal of Computing Physics 1994; 115:200-212.

3. Jiang GS, Shu CW. Efficient implementation of weighted ENO schemes. Journal of Computing Physics 1996; 126:202-228.

4. Pirozzoli S. Numerical methods for high-speed flows. Annual Review Fluid Mechanics 2011; 43:94-163.

5. Suresh A, Huynh HT. Accurate monotonicity-preserving schemes with Runge-Kutta time stepping. Journal of Computing Physics 1997; 136:83-99. 
6. Adams NA, Shariff K. A high-resolution hybrid compact-ENO scheme for shock-turbulence interaction problems. Journal of Computing Physics 1996; 127:27-51.

7. Kim D, Kwon HK. A high-order accurate hybrid scheme using a central flux scheme and a WENO scheme for compressible flow field analysis. Journal of Computing Physics 2005; 210:554-583.

8. Pirozzoli S. Conservative hybrid compact-WENO schemes for shock-turbulence interaction. Journal of Computing Physics 2002; 178:81-117.

9. Ren YX, Liu M, Zhang H. A characteristic-wise hybrid compact-WENO scheme for solving hyperbolic conservation laws. Journal of Computing Physics 2003; 192:365-386.

10. Sun ZS, Ren YX, Larricq C, Zhang SY, Yang SY. A class of finite difference schemes with low dispersion and controllable dissipation for DNS of compressible turbulence. Journal of Computing Physics 2011; 230(12):4616-4635.

11. Martín MP, Taylor EM, Wu M, Weirs VG. A bandwidth-optimized WENO scheme for effective direct numerical simulation of compressible turbulence. Journal of Computing Physics 2006; 220:270-289.

12. Wang ZJ, Chen RF. Optimized Weighted essentially nonoscillatory schemes for linear waves with discontinuity. Journal of Computing Physics 2001; 174:381-404.

13. Lele SK. Compact finite difference schemes with spectral-like resolution. Journal of Computing Physics 1992; 103: $16-42$.

14. Tam CKW, Webb JC. Dispersion-relation-preserving finite difference schemes for computational acoustics. Journal of Computing Physics 1993; 107:262-281.

15. Pirozzoli S. On the spectral properties of shock-capturing schemes. Journal of Computing Physics 2006; 219: 489-497.

16. Steger JL, Warming RF. Flux vector splitting of the inviscid gasdynamic equations with applications to finite difference methods. Journal of Computing Physics 1981; 40:263-293.

17. Van Leer B. Flux-vector splitting for the Euler equations. In Technical Report ICASE. NASA Langley Research Center: USA, 1982; 82-30.

18. Roe PL. Approximate Riemann solvers, parameter vectors and difference schemes. Journal of Computing Physics 1981; 43:357-372.

19. Shu CW, Dinshaw SB. Monotonicity preserving weighted essentially non-oscillatory schemes with increasingly high order of accuracy. Journal of Computing Physics 2000; 160:405-452.

20. Shu CW, Osher S. Efficient implementation of essentially non-oscillatory shock-wave schemes. II Journal of Computing Physics 1989; 83:32-78.

21. Woodward P, Colella P. The numerical simulation of two-dimensional fluid flow with strong shocks. Journal of Computing Physics 1984; 54:115-173.

22. Shi J, Zhang YT, Shu CW. Resolution of high order WENO schemes for complicated flow structures. Journal of Computing Physics 2003; 186:690-696.

23. Samtaney R, Pullin DI, Kosovi B. Direct numerical simulation of decaying compressible turbulence and shocklet statistics. Physics of Fluids 2001; 13:1415-1430. 